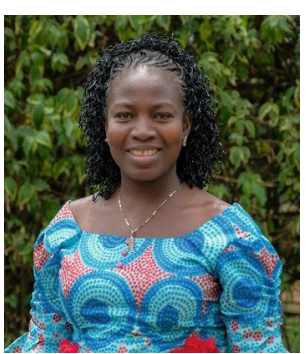

Credit: Target Malaria,

Imperial College London

\title{
We need to redefine the relationship between science and its end-users
}

\author{
Scientific research can have a positive impact on society, particularly in a health crisis. But \\ to fully achieve this, scientists must engage with end-users from the very beginning of the \\ research process, writes Lea Pare Toe.
}

$\mathrm{S}$ cience makes a fundamental contribution to society on many levels; it seems to be one of humankind's greatest enterprises in the interest of human welfare. Yet this does not exempt it from challenges regarding its perception, and scepticism over scientific findings and arguments is certainly not new. The COVID-19 pandemic has revealed how people will deny scientific arguments to the extent of questioning the very existence of a virus or its severity. While scientists have advanced the evidence for transmission and deadliness, ordinary citizens-including leaders of countries-have notably refrained from applying scientific recommendations to control the disease.

The situation is similar in my field of research, malaria: while scientists and funders put time, money and effort into tools to counter the disease, communites are slow to adopt them. Tension therefore exists between the ambition of the scientists to manage the disease, on the one hand, and denial of the science or non-application of the recommended measures by end-users, on the other. Politico-economic or cultural issues may explain this resistance, but more important perhaps is the absence of involvement of the end users in the actual process of research.

From an ethical and legal perspective, there exists robust guidance for responsible research. What is lacking, though, is a sense of the importance of community engagement in a research process to help build wider knowledge and confidence.

Indeed, the ethical guidelines themselves are not always well understood: informed consent does not necessarily guarantee comprehension of the research's purpose, and signing a consent form is not in itself evidence for this. In a clinical trial involving 440 children in Burkina Faso (https://doi. org/10.1371/journal.pone.0080800), more than $70 \%$ of the parents opted for their children to participate prior to knowing the details thought to be necessary for informed consent. An informed consent process equates neither to two-way communication nor to active collaboration. And participatory research as a collaborative effort-designed to ensure that research activities are responding to the needs of a host community-only reaches its goal at the end of the research, when the findings are available. Such considerations suggest that even where efforts are made to establish understanding between scientists and end users, from the community's standpoint this can be passive rather than active in essence.

With this in mind, there is a distinct argument for redefining the way we do science and particularly the level of end-user involvement in the research process. Both sides need to be close and proactive in establishing a two-way platform for communication. At an early-stage, scientists may communicate goals, processes, expectations and constraints, while end-users may feedback their own hopes and anxieties. On this basis, the final outcome becomes something owned by both sides and treated accordingly.

One of the reasons often used to justify the passive partnership between researchers and end-users is that the research is too complex or a research programme carries uncertainties and is therefore difficult to communicate.

In recent years, an alternative approach has emerged in the form of stakeholder engagement. Stakeholder engagement is defined as dialogue between the research team and end-users, and it involves exchange of ideas as well as mutual respect for the values and interests on both sides. This fosters active involvement of the stakeholders in the research process. Engagement should begin early and continue across the research timeline. Bringing end-users into the research pathway generates a strong level of commitment to scientific process and findings. But how can we bring a general audience on board in such a specialized field?
I believe that there are two important prerequisites for achieving this. First, as scientists, we must adjust our mind-sets and acknowledge the need to involve end-users in the research process. Second, we have to find appropriate ways to build non-scientists' understanding by communicating scientific knowledge more effectively.

In the case of our experience with Target Malaria (https://www.targetmalaria. org), we have come to understand that a wider audience can indeed understand the complexities of the science if communication is carefully thought through. Target Malaria is a consortium for research on genetically modified mosquitoes to control malaria vectors in Africa. Here research teams and hosting communities co-developed the research process by designing a communication model to facilitate the understanding of essential concepts related to genetic modification (DNA, genes, etc.). This led to the empowerment of the community, who could then voice their views, and provided knowledge for informed decision-making on the science.

Alongside co-development, community feedback was used to improve and refine the research process. For example, stakeholder questions in relation to the possible transfer of sterility in the genetically modified mosquito into the human population led our consortium to consider this in their risk analysis. In the same vein, our consortium launched a study on the ecological interactions of the communities around Anopheles gambiae's larval and adult niches, including food-webs and pollination interactions. But the most valuable outcome of this two-way dialogue was the end-users' understanding of such a complex, specialist research project and their commitment to the research process.

Overall, redefining the relationship between scientists and end-users would 
seem now to be urgent for improving the way scientific evidence is more generally accepted, particularly in a health crisis such as the COVID-19 pandemic.
Lea Pare Toe ${ }^{凶}$

Institut de Recherche en Sciences de la Santé, Bobo-Dioulasso, Burkina Faso.

․e-mail:lea_toe@yahoo.com
Published online: 21 December 2020

https://doi.org/10.1038/s41562-020-01023-2

Competing interests

The author declares no competing interests 